\title{
The Wheat Plastochron Mutant, fushi-darake, Shows Transformation of Reproductive Spikelet Meristem into Vegetative Shoot Meristem
}

\author{
Chikako Tahira $^{1}$, Naoki Shitsukawa ${ }^{1}$, Yusuke Kazama ${ }^{2}$, Tomoko Abe ${ }^{3}$, Koji Murai ${ }^{*}$ \\ ${ }^{1}$ Department of Bioscience, Fukui Prefectural University, Eiheiji-cho, Japan; ${ }^{2}$ RIKEN Innovation Center, Woko, Japan; ${ }^{3}$ RIKEN \\ Nishina Center, Woko, Japan. \\ Email: ${ }^{*}$ murai@fpu.ac.jp
}

Received October $7^{\text {th }}, 2013$; revised October $30^{\text {th }}, 2013$; accepted November $7^{\text {th }}, 2013$

Copyright (C) 2013 Chikako Tahira et al. This is an open access article distributed under the Creative Commons Attribution License, which permits unrestricted use, distribution, and reproduction in any medium, provided the original work is properly cited.

\begin{abstract}
In wheat plants at the vegetative growth stage, the shoot apical meristem (SAM) produces leaf primordia. When reproductive growth is initiated, the SAM forms an inflorescence meristem (IM) that differentiates a series of spikelet meristem (SM) as the branch. The SM then produces a series of floret meristem (FM) as the branch. To identify the mechanisms that regulate formation of the reproductive meristems in wheat, we have investigated a leaf initiation mutant, fushi-darake (fdk) which was developed by ion beam mutagenesis. The morphological traits were compared in wild type (WT) and fdk mutant plants grown in the experimental field. WT plants initiated leaves from SAM at regular intervals in spiral phyllotaxy, while $f d k$ plants had $1 / 2$ alternate phyllotaxy with rapid leaf emergence. The $f d k$ plants have increased numbers of nodes and leaves compared with WT plants. The time interval between successive leaf initiation events (plastochron) was measured in plants grown in a growth chamber. The fdk plants clearly show the rapid leaf emergence, indicating a shortened plastochron. Each tiller in fdk plants branches at the upper part of the culm. The fine structure of organ formation in meristems of $f d k$ plants was examined by scanning electron microscopy (SEM). The SEM analysis indicated that $f d k$ plants show transformation of spikelet meristems into vegetative shoot meristems. In conclusion, the $f d k$ mutant has a heterochronic nature, i.e., both reproductive and vegetative programs were simultaneously in operation during the reproductive phase, resulting in a shortened plastochron and transformation of reproductive spikelets into vegetative shoots.
\end{abstract}

Keywords: Einkorn Wheat; Heterochrony; Ion Beam Mutagenesis; Phyllotaxy; Plastochron; Shoot Meristems; Spikelet Meristems; Triticum monococcum

\section{Introduction}

Grasses such as wheat (Triticum aestivum) are the major source of carbohydrates for humans, and the yield of grain from these crops is largely dependent on inflorescence architecture. A detailed understanding of development in wheat plants is of value not only to wheat breeding but also for basic scientific research. In its vegetative growth phase, a wheat plant is composed of roots, stems, and leaves. Leaf initiation occurs at the base of the shoot apical meristem (SAM), a dome of cells, located at the tip of the shoot. In wheat, one to three leaf primordia are initiated during embryogenesis. After seedling emergence, the SAM produces five to seven leaf primordia; the ac-

\footnotetext{
"Corresponding author.
}

tual number produced varies with genotype and environmental conditions, such as vernalization and photoperiod [1]. The SAM gradually elongates and produces leaf primordia as single ridges until the phase transition from vegetative to reproductive growth [2]. The pattern of formation of leaf primordia around the stem, termed phyllotaxy, is very precisely controlled and is characteristic for the plant species [3]. The wheat plant shows spiral phyllotaxy. The time interval between successive leaf primordium initiations is referred to as the plastochron. The plastochron also depends on genotype and environmental conditions. When the plant transits from the vegetative to reproductive growth phase, the SAM ceases production of leaf primordia, and thus the maximum number of leaves is determined at this point. At phase 
transition, the elongated SAM is transformed into an inflorescence meristem (IM), i.e., spike primordia, with double ridges: the lower ridge is a leaf primordium that does not develop further, and the upper ridge becomes a spikelet primordium [4]. The spikelet is a reproductive unit unique to the grass inflorescence $[5,6]$. The wheat spikelet is composed of florets and is enclosed by two small bract leaves (called glumes in wheat) [7]. Spikelet meristems (SMs) are arranged in two alternate vertical ranks along the length of the inflorescence. The initiation of spikelet primordia continues until formation of the terminal spikelet in the apical meristem. The number of spikelets per spike depends on genotype and environmental conditions. After the initiation of spikelet primordia, floret initiation begins in each spikelet. In bread wheat, usually four to five floret primordia are initiated from each floret meristem (FM) in each spikelet [2]. The fertile floral organs, such as pistil and stamens, develop within each floret [7].

In adult wheat plants, the stem or culm of each tiller is cylindrical and consists of hollow internodes separated by solid nodes. About five nodes are usually visible, and the internodes at higher position are longer than those at lower. The length of the internodes depends on genotype and environmental conditions. Each leaf consists of a sheath, ligule, auricles and blade. The leaf sheath attaches to the stem at the node; the structural unit composed of a leaf joined to a stem node is called a phytomer (Figure 1). Typically, a phytomer has an associated axillary bud in the leaf axil. In the lower part of the culm, phytomers are not elongated and produce tillers from the axillary bud. The elongated phytomers in the upper part of the culm do not produce tillers.

After the flag leaf unfolds, the spike (inflorescence) appears from the tip of the culm (Figure 1). The spikelets are arranged as two opposite rows on the main axis (the rachis). The number of spikelets per spike is determined by the timing of terminal spikelet initiation. In this respect, the rachis meristem (inflorescence meristem) in wheat is determinate [2]. The spikelet is composed of florets joined at the axis (rachilla) alternately on opposite sides, and encompassed by two bract leaf-like glumes. There are multiple florets in each spikelet. The maximum number of florets per spikelet also depends on genotype and environmental conditions. In contrast to barley, rice and maize, the wheat rachilla meristem is classified as indeterminate [2]. In each floret, the reproductive organs are enveloped by two leaf-like structures, a lemma and a palea. The lemma and palea correspond to the bract, but their origins are still unclear. An individual wheat flower contains one pistil, three stamens and two lodicules [7]. The lodicules are formed on the lemma side and are considered to be analogous to the petal of other angiosperms.

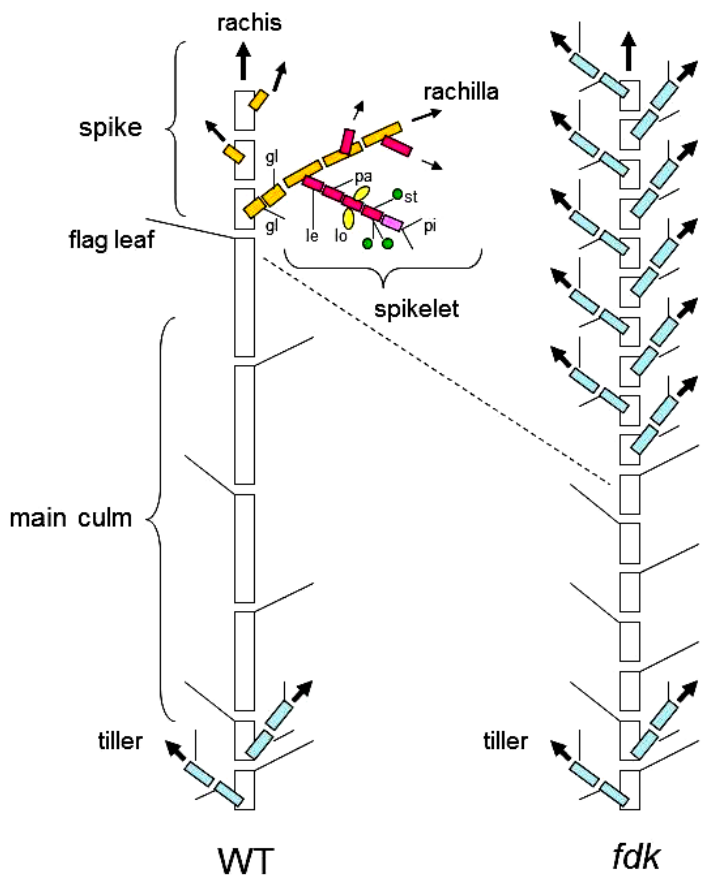

Figure 1. Schematic illustrations of phytomeric structures in wild-type (WT) and fushi-darake (fdk) mutant plants at maturing stage. (Left) The phytomeric structure of a WT plant. The culm consists of elongated phytomers with spiral phyllotaxy. The spikelets are arranged as two opposite rows of lateral branches from the main axis (rachis). Each spikelet is composed of florets, joined at the axis (rachilla) alternately on opposite sides, enclosed by two glumes. Each floret is composed of a lemma, a palea, two lodicules, three stamens and a pistil. (Right) The phytomeric structure of a fdk plant. Short phytomers with alternate phyllotaxy emerge rapidly. Each spikelet is converted into a vegetative shoot. gl, glume; le, lemma; pa, palea; lo, lodicule; st, stamen; pi, pistil.

The inflorescence architecture is species-specific in grasses and is determined by the particular arrangement of the spikelets. Several genes that control spikelet development have been identified in grasses such as rice and maize. The inflorescences of maize and rice are more complicated than that of wheat because of the presence of additional axillary branch meristems: the tassel branch and spikelet pair meristem in maize, and the panicle branch meristem in rice $[5,8]$. In rice, ABERRANT PANICLE ORGANIZATION 1 (APO1), OSMADS34/ PANICLE PHYTOMER 2 (PAP2), and APO2/Rice FLORICAULA (RFL) have been demonstrated to control the initiation of the transition from branch meristems (BMs) to spikelet meristems (SMs). APO1 encodes an F-box protein orthologous to Arabidopsis UNUSUAL FLORAL ORGANS (UFO) and suppresses precocious conversion of BM to SM [9-11]. OsMADS34/PAP2 is a member of the SEPALLATA (SEP) subfamily of MADS-box genes, and positively regulates SM identity $[12,13]$. Further- 
more, $A P O 2 / R F L$ is a rice ortholog of Arabidopsis $L E A F Y$, and suppresses transition into SM through interaction with APO1 [14].

\section{Materials and Methods}

\subsection{Plant Material}

Seeds of the diploid einkorn wheat (Triticum monococcum, genome constitution AA, $2 n=2 x=14$ ) strain KU104-1 were given 50 Gy of nitrogen ion beam treatment and then sown in the field. Ion beam treatment, which has a high linear energy transfer (LET), has been demonstrated previously to be an efficient inducer of mutations in plants and to predominantly cause null mutations $[15,16]$. The spikes of $\mathrm{M}_{1}$ plants were bagged and the harvested selfed seeds of each spike were used to produce the $\mathrm{M}_{2}$ lines. From approximately $1,200 \mathrm{M}_{2}$ lines, we identified a mutation that had an abnormally large number of nodes; we termed this mutation fushi-darake $(f d k)$, which means too many nodes in Japanese. Wild type (WT) strain KU104-1 and $\mathrm{M}_{2} f d k$ plants were used in the experiments.

\subsection{Growth Condition}

For the morphological study, WT and $f d k$ plants were grown in the experimental field of Fukui Prefectural University. In the field, seeds were sown in mid-October and WT plants were harvested in the following June. Although $f d k$ plants can have a small spike in the main tiller, they are generally sterile. For the examination of leaf number and leaf initiation speed (plastochron), the WT and fdk plants were grown in a growth chamber under long-day $\left(16 \mathrm{~h}\right.$ light $/ 8 \mathrm{~h}$ dark) conditions at $20^{\circ} \mathrm{C}$ $\left(100 \mu \mathrm{E} \cdot \mathrm{m}^{-2} \cdot \mathrm{s}^{-1}\right)$.

\subsection{Examination of Morphological Phenotypes and Plastochron}

Three WT and four $f d k$ plants grown in the field were used for examination of culm and internode lengths. WT plants usually produce spikes in each tiller, whereas the fdk plants only occasionally produce a single spike in the main tiller. At the maturing stage, WT plants in the field were sampled and internode and spike lengths were measured. The $f d k$ plants mature later than WT plants, and stop growing in the field. The $f d k$ plants were then also sampled at the maturing stage. Thirteen WT plants and three $f d k$ plants grown in a growth chamber were examined for the timing of leaf unfolding. Days from germination to leaf unfolding were identified and plotted.

\subsection{Scanning Electron Microscopy (SEM)}

The structure of the spikes of $f d k$ plants was investigated using scanning electron microscope (SEM). A low-vacuum SEM (S-3000N, Hitachi Co., Ltd., Tokyo, Japan) was used to observe the morphological features of inflorescences. Young spikes were frozen at $-15^{\circ} \mathrm{C}$ on a cool stage and examined under low vacuum conditions $(30 \mathrm{~Pa})$ with an accelerating voltage of 15 or $20 \mathrm{kV}$.

\section{Results}

\subsection{Morphological Characteristics of the fushi-darake Mutant}

The fushi-darake (fdk) mutant plant has been identified during the development of a large-scale mutant panel developed using heavy-ion beam mutagenesis [17]. The $f d k$ showed drastic changes to their structural organization compared to wild type (WT) plants in the field (Figure 2A). Immediately after germination, $f d k$ and WT seedlings were indistinguishable. However, WT plants initiated leaves from the shoot apical meristem (SAM) at regular intervals in spiral phyllotaxy. By contrast, $f d k$ plants had $1 / 2$ alternate phyllotaxy with rapid leaf emergence (Figure 2B). Consequently, the $f d k$ plants had a larger number of nodes and leaves compared to the WT plants. Furthermore, the leaves of $f d k$ plants were smaller than those of WT plants with respect to blade and sheath lengths and leaf blade widths (Figure 2B). In the $f d k$ plants, vegetative shoot branches emerged from the nodes in the upper part of the culm of most tillers (Figure 2C). In these ectopic shoots, normal leaves were produced with $1 / 2$ alternate phyllotaxy. Each tiller had zero to nine branches (Table 1). The culms of fdk plants were unable to support the heavy upper vegetative shoots, with the result that the plants collapsed onto the ground (Figure 2A).

We compared the number of nodes and the internode lengths in WT and $f d k$ plants grown in the field (Figure 3). In WT plants, only the upper five internodes elongated substantially at the transition from vegetative to reproductive growth. We designated node position numbers as follows: the uppermost node was designated as node 1 , and then nodes $2,3,4,5$ and 6 were counted from the top. The spike is attached at node 1 in the WT plants. The internodes were designated in a similar fashion as the nodes: the internode just below node 1 was designated as internode- 1 , and then internodes- $2,-3,-4$, and -5 were counted in sequence from the top. Internode1 was the longest, with internode length decreasing from upper to lower: each internode was approximately twice as long as the next lower one (Figure 3). Compared with WT plants, $f d k$ plants were smaller and showed an increased number and abnormal pattern of elongated internodes. Unlike WT plants with six nodes, $f d k$ plants had 19 to 20 nodes, and each internode length was almost identical. 
Reproductive Spikelet Meristem into Vegetative Shoot Meristem

Table 1. Phenotypic difference between the wild-type (WT) and fushi-darake (fdk) plants grown in the field.

\begin{tabular}{|c|c|c|c|c|c|c|}
\hline Line & $\begin{array}{l}\text { Total no. } \\
\text { tillers }\end{array}$ & $\begin{array}{l}\text { No. of tillers } \\
\text { with spike }\end{array}$ & $\begin{array}{l}\text { Spike length } \\
(\mathrm{cm})\end{array}$ & $\begin{array}{c}\text { No. of spikelets } \\
\text { per spike }\end{array}$ & $\begin{array}{c}\text { No. of } \\
\text { branched tillers }\end{array}$ & $\begin{array}{c}\text { Average (min.-max.) } \\
\text { no. of branches }\end{array}$ \\
\hline WT \#1 & 16 & 16 & 7.2 & 32 & $\mathrm{nd}^{\mathrm{a}}$ & $\mathrm{nd}^{\mathrm{a}}$ \\
\hline WT \#2 & 20 & 20 & 8.4 & 34 & $\mathrm{nd}^{\mathrm{a}}$ & $\mathrm{nd}^{\mathrm{a}}$ \\
\hline WT \#3 & 23 & 23 & 8.2 & 32 & $\mathrm{nd}^{\mathrm{a}}$ & $\mathrm{nd}^{\mathrm{a}}$ \\
\hline$f d k \# 1$ & 13 & 1 & 2.9 & 10 & 13 & $4.1(2-9)$ \\
\hline$f d k \# 2$ & 13 & 1 & 2.3 & 12 & 12 & $4.4(0-8)$ \\
\hline$f d k \# 3$ & 15 & 1 & 2.2 & 11 & 15 & $3.7(2-7)$ \\
\hline
\end{tabular}

a not detected.

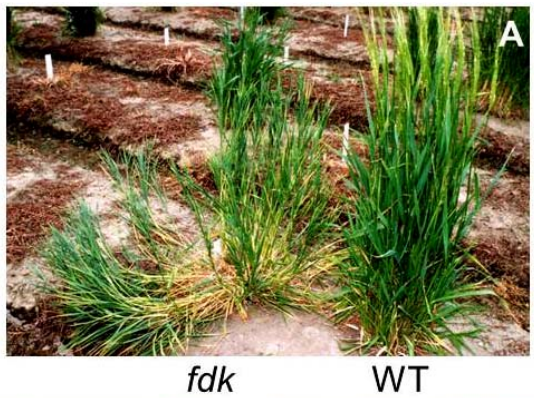

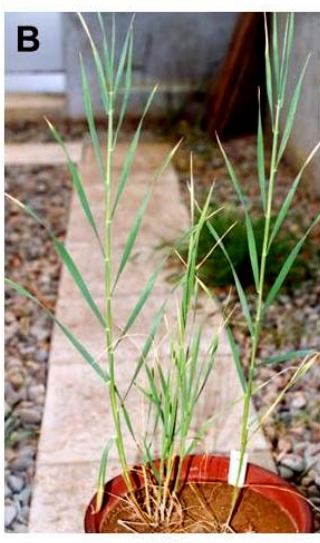

$f d k$

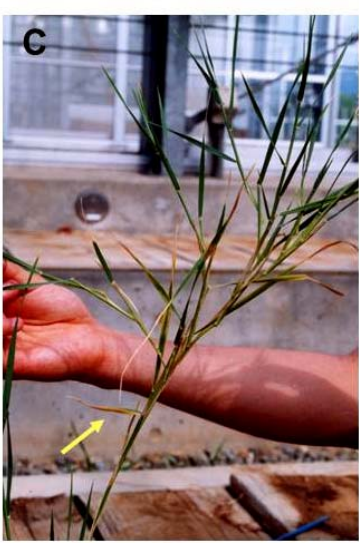

$f d k$
Figure 2. Phenotypes of WT and $f d k$ plants. (A) WT and $f d k$ plants grown in the experimental field. The WT plants are at the pollination stage. (B) Young fdk plants showing 1/2 alternate phyllotaxy with rapid leaf emergence. (C) Adult $f d k$ plant with ectopic vegetative shoots as branches in the upper part of the main culm. The leaf corresponding to the flag leaf of WT plants is indicated by an arrow.

The uppermost internodes on the main tiller of $f d k$ plants did not elongate. A small spike was present at the tip of culm (Figure 4A). The spikes averaged approximately $8 \mathrm{~cm}$ in length in WT plants and consisted of 32 to 34 spikelets (Table 1). In contrast to WT plants, the spikes of $f d k$ plants were small $(2-3 \mathrm{~cm})$ and contained fewer spikelets $(10-12)$. The florets of $f d k$ plants contained a developing pistil and stamens (Figure 4B). However, the stamens were wrinkled and immature, re-

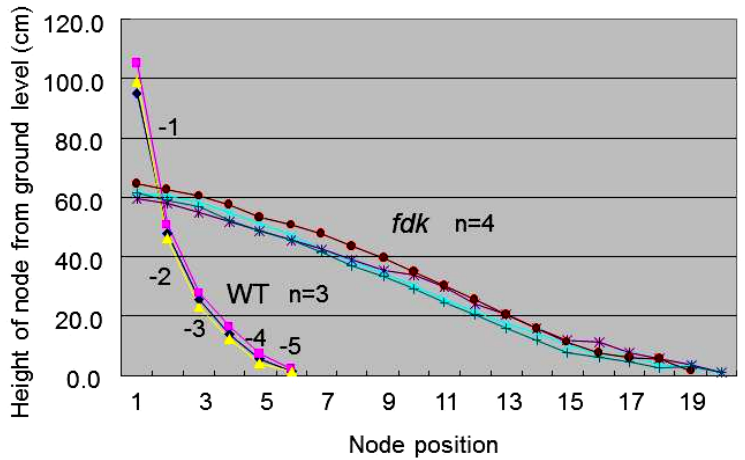

Figure 3. Internode elongation patterns in WT and $f d k$ plants. Nodes are numbered from the uppermost one downwards. Internode numbers are also indicated. Plants were sampled at maturing stage.
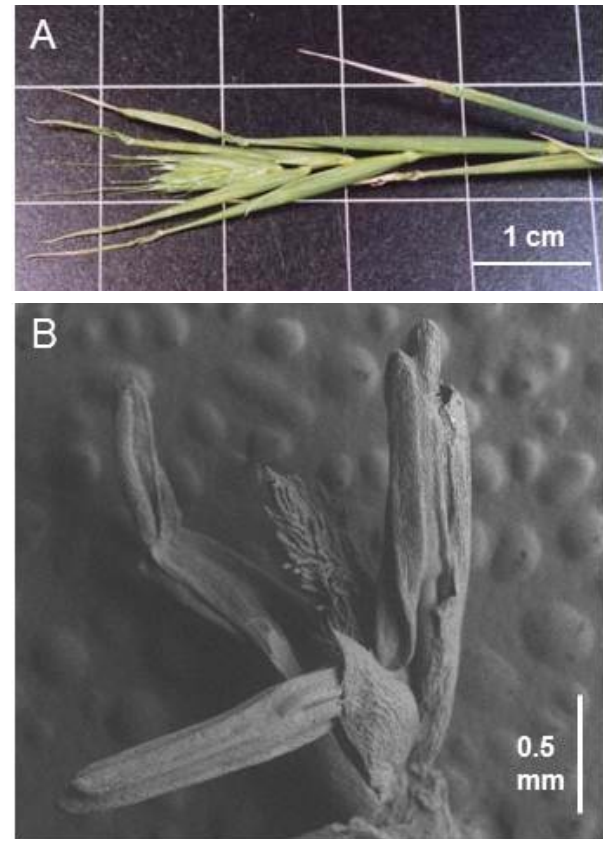

Figure 4. Small spike and floral organs at the tip of the main culm of $f d k$ plants. (A) Small spike of the fdk plant. (B) Scanning electron microscope (SEM) image of the floral organs in the floret of the small spike. 
sulting in sterility and a failure of seed set in $f d k$ plants.

\subsection{Plastochron of $\boldsymbol{f d k}$ Mutants}

The fdk mutant seedlings could be distinguished from WT by the rapid emergence of leaves. We examined the timing of leaf unfolding in WT and $f d k$ seedlings grown in a growth chamber (Figure 5). It took about 70 days to unfold nine leaves in the WT plants. In contrast to WT plants, $f d k$ plants produced approximately 18 leaves over a period of 70 days. The rate of leaf emergence of the first three leaves between WT and $f d k$ was similar. However, the rate of leaf emergence was more rapid in $f d k$ compared to WT after the 3-leaf stage. This indicates that rapid leaf emergence in $f d k$ resulted from a rapid rate of leaf initiation: the plastochron of $f d k$ plants was estimated to be half of that in WT (Figure 5). In WT plants, the emergence of successive leaves ceased following the initiation of the flag leaf, which could be distinguished from the other leaves by its short blade and the emergence of a spike from its leaf sheath. The $f d k$ plants did not have a typical flag leaf below the small spike (Figure 4A).

\subsection{SEM Analysis of Shoot Apical Meristem Development in $f d k$ Plants}

To investigate further the morphological differences between WT and $f d k$ plants, we carried out an SEM analysis of shoot apical meristem (SAM) development (Figures 6 and 7). The initial steps of SAM development were similar in both WT and $f d k$ plants (Figures 6A and 7A). After transition from the vegetative to reproductive phase in WT plants, the SAM elongated and transformed into an inflorescence meristem (IM). The IM formed spikelet meristems (SMs) in two opposite rows, and a pair of glumes was differentiated as the first organ of the spikelet (Figure 6B). At the floret differentiation stage, the SM produced the floret meristem (FM) as a lateral branch (Figures 6C, D). Four or five floret primordia were usually initiated in the spikelets of the WT plants, and one or two florets developed floral organs, such as a pistil and stamens (Figure 6E).

SAM development in $f d k$ plants was very different to that of WT plants. The SAM elongated but its branch meristems (BMs) resembled leaf primordia rather than spikelet meristems (SMs) (Figure 7B). Ectopic BMs that were similar to leaf primordia were initiated with a $1 / 2$ alternate pattern. Later, the elongated SAM produced flat dome-like BMs (Figures 7C and D). These observations, together with those on the morphology of the $f d k$ mutants (Figure 2), suggest that the flat dome-like BMs develop into vegetative shoots. Several spikelet primordia with floret meristems (FMs) were differentiated ultimately at the tip of the SAM (Figure 7E).

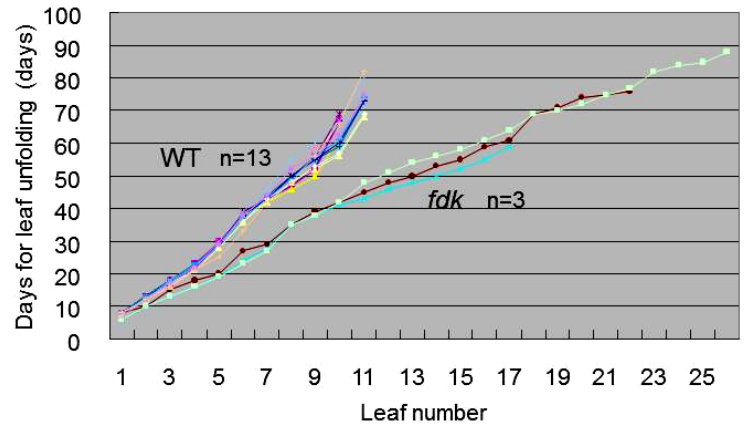

Figure 5. Increase in the number of leaves during development in WT and $f d k$ plants. The day of unfolding of each leaf is indicated.
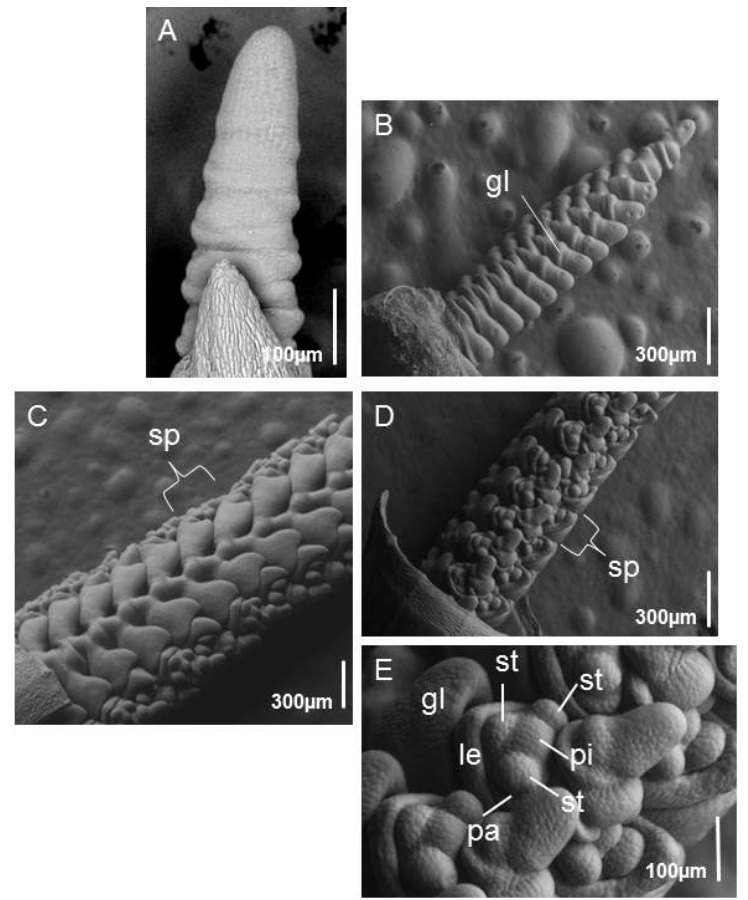

Figure 6. SEM images of the shoot apical meristem (SAM) and spike development in WT plants. (A) The SAM just before phase transition from vegetative to reproductive growth. (B) Young spike at the spikelet differentiation stage. Glume primordia initiate in the spikelet in the middle section of the spike. (C) Side view of a spike at the floret differentiation stage. A spikelet is indicated. (D) Front view of a spike at the floret differentiation stage. A spikelet is indicated. (E) Higher magnification image of florets. sp, spikelet, gl, glume; le, lemma; pa, palea; lo, lodicule; st, stamen; pi, pistil.

\section{Discussion}

\subsection{The fdk Is a Wheat Plastochron Mutant}

The time interval between successive leaf initiations (the plastochron) varies among plant species, and is also affected by the age of the plants and by environmental conditions [3]. Our analysis here of the fdk mutant of 

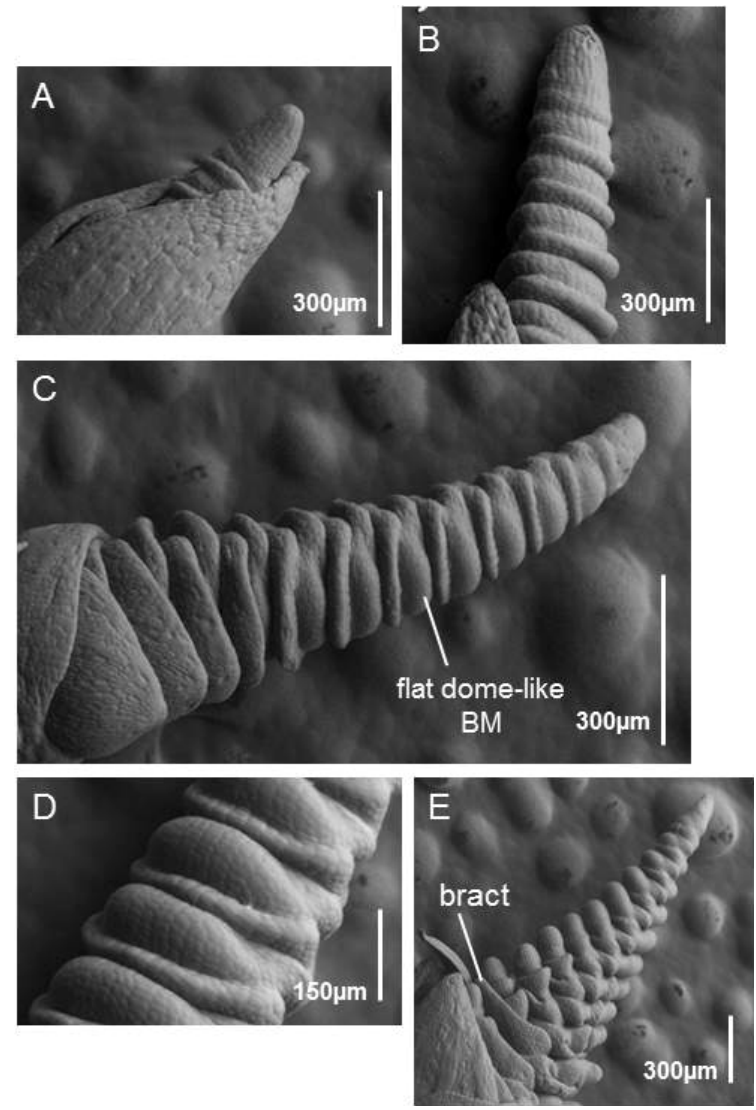

Figure 7. SEM images of SAM development in $f d k$ plants. (A) The vegetative SAM. (B) The elongated SAM. Leaf primordia with alternate phyllotaxy are differentiated instead of spikelet primordia. (C) A late development stage of an elongated SAM. The flat dome-like primordia have differentiated into vegetative shoot meristems instead of spikelet meristems. (D) Higher magnification image of flat dome-like meristems. (E) Spikelets eventually differentiate at the tip of the elongated SAM. The elongated bract-like leaf is initiated above each spikelet.

einkorn wheat has shown that it has a shortened plastochron (Figures 2 and 5). Three plastochron mutants, plastochron 1 (pla1), pla2 and pla3, have been identified in rice (Oryza sativa) [18-20]. The pla1 mutant of rice has a plastochron that is reduced to approximately half that of the WT, and thus somewhat similar to the $f d k$ mutant [18]. The WT gene, PLASTOCHRON 1 (PLA1), encodes a member of a plant-specific subfamily of cytochrome P450, CYP78A11, which potentially catalyzes substances controlling plant development [21]. PLA1 is expressed in developing leaf primordia but not in the SAMs, suggesting that it may have a non-cell-autonomous influence on the SAMs of developing leaf primordia to affect the timing of successive leaf initiation. The phenotype of the pla2 mutant is similar to that of pla 1 , although its plastochron is significantly shorter [19]. The WT PLA2 gene is an ortholog of maize (Zea mays) ter- minal ear 1 (te1), which encodes a MEI2-like RNA binding protein [19]. The maize te 1 mutant has a shortened plastochron with abnormal phyllotaxy [22]. PLA2 is also expressed in leaf primordia but may act independently of PLA1 to inhibit leaf initiation. Plants carrying both pla1 and pla2 show a synergistic phenotype, with more rapid initiation of leaves and a plastochron that is even shorter than either single mutant [19]. The pla3 mutation has been shown to be allelic to goliath (go) [20]. $P L A 3 / G O$, which encodes a glutamate carboxypeptidase, is an ortholog of Arabidopsis ALTERED MERISTEM PROGRAM 1 (AMP1) [23]. In addition to the shortened plastochron, pla3 showed pleiotropic effects including an enlarged embryo, seed vivipary, defects in SAM maintenance and aberrant leaf morphology [20]. PLA3 is expressed in the whole plant body and seems to be involved in plant hormone homeostasis. The plastochron of pla3 is shorter than those of pla1 and pla2. The plastochron of wheat $f d k$ mutant is reduced to half that of the WT plants (Figure 5) and is therefore affected to a similar extent as rice pla 1 rather than either of the other pla mutants; this suggests the possibility that WT FDK functions in the same metabolic pathway as PLA1.

\subsection{The fdk Shows Transformation of Spikelets into Vegetative Shoots}

The inflorescence of grass species is composed of a unique unit called the spikelet. When the wheat plant transits from the vegetative to reproductive growth phase (flowering), the SAMs are elongated and spikelet meristems (SMs) initiate as lateral branches (Figure 6). Our SEM analysis of the $f d k$ mutant indicated that differentiation of SMs was delayed and the leaf primordia were initiated from branch meristems (BMs) with 1/2 alternate phyllotaxy (Figure 7B). These observations suggest that $1 / 2$ alternate phyllotaxy with rapid leaf emergence produced the shortened plastochron in the $f d k$ mutant. The SAMs further elongated and produced flat dome-like BMs at the position of the original SMs (Figures 7C and D). We also found that $f d k$ plants had vegetative shoot branches emerging from the nodes of upper part of culm of almost all tillers (Figure 2C). Thus, our results suggest that these vegetative shoots are likely to be developed from the BMs of elongated SAMs. In conclusion, our findings indicate that the abnormal phenotype of the $f d k$ mutant resulted from transformation of SMs into vegetative shoots (Figure 1). Assuming that the ectopic vegetative shoots in the upper part of the culm are caused by transformation of spikelets in the $f d k$ mutant, then the lowest node with a branched ectopic shoot corresponds to the spike neck of WT plants. According to this interpretation, the leaf just below the lowest branching node should correspond to the original flag leaf (Figure 2A). 
The duration of the leaf emergence phase, from germination through to flag leaf unfolding, was almost identical in $f d k$ and WT plants (Figure 5). Thus, the $f d k$ mutant did not affect the timing of flowering, although the number of phytomers was doubled.

The genetic mechanism for spikelet development in rice can be divided into two aspects: first, the initiation of SM identity; and second, maintenance of SM identity. The initiation of SMs is controlled by ABERRANT PANICLE ORGANIZATION 1 (APO1), which encodes an UNUSUAL FLORAL ORGANS (UFO)-like F-box protein, OSMADS34/PANICLE PHYTOMER 2 (PAP2), which encodes a SEPALLATA (SEP)-like MADS-box transcription factor, and APO2/Rice FLORICAULA (RFL), which encodes a $L E A F Y$ ( $L F Y)$-like protein [9-14]. The maintenance of SM identity in rice is regulated by the action of FRIZZY PANICLE (FZP1) [24], which belongs to the APETALA2 (AP2) transcription factor family; knockout mutants of FZP1 exhibit defects in the FM and branch structures at the floret position. The wheat orthologs of SEP, LFY and AP2 have been identified [8, 25,26]. The expression pattern of the wheat $L F Y$ ortholog, Wheat FLORICAULA (WFL), indicated that this gene is associated with spikelet initiation [8]. Investigation of the relationship between the fdk phenotype and WFL could provide insights into the pathways adversely affected by the fdk mutation.

\subsection{Comparison of Inflorescence Structures in Wheat $f d k$ and Rice pla Mutants}

In wheat, the spikelets are initiated as the primary branch of the main axis, the rachis [7]. By contrast, in rice, the primary branch meristems (BMs) are developed from inflorescence meristems (IMs) as axillary organs, and secondary BMs are produced from the primary BMs as axillary meristems [27]. The spikelets are initiated from secondary-branch or directly from primary branch.

In rice pla 1 mutants, after the unfolding of the flag leaf, several vegetative shoots are produced instead of a panicle [18]. The ectopic shoots showed spiral phyllotaxy instead of the $1 / 2$ alternate pattern. In the nodes below the flag leaf in both pla1 and WT plants, a 1/2 alternate phyllotaxy pattern was present. Spiral phyllotaxy is observed in WT plants only after differentiation of the primary BMs of the panicle. In each of these ectopic shoots, normal leaves are produced with alternate phyllotaxy. Thus, the rachis primary branches are converted into vegetative shoots [18]. The rice pla 2 and pla 3 mutants also produce ectopic shoots instead of a panicle $[19,20]$. The number of ectopic shoots in pla3 is larger than in pla 1 and pla2. The $f d k$ mutant exhibited a similar phenotype to the rice pla mutants with regard to conversion of the reproductive branch into vegetative shoots. Our SEM analysis indicated that SMs, as branches of the rachis, converted into vegetative shoots (Figures 7C and D). By contrast to rice, the wheat primary BMs $(=\mathrm{SMs})$ show $1 / 2$ alternate phyllotaxy, resulting in ectopic shoots that exhibit alternate phyllotaxy. In the fdk mutant, alternate phyllotaxy is shown in the nodes below the flag leaf rather than the spiral phyllotaxy present in WT plants (Figure 2B). Our SEM analysis indicated that elongated SAMs initiate leaf primordia with alternate phyllotaxy (Figure 7B). Elongated SAMs are usually observed in IMs during the transit into the reproductive growth phase. These observations indicate that in the fdk mutant, both vegetative and reproductive programs are simultaneously expressed. However, the duration of the period from germination to flag leaf initiation is similar in $f d k$ and WT plants (Figure 5), indicating that acquisition of flowering competency is not prolonged [28]. The heterochronic nature of the $f d k$ mutant, i.e., the simultaneous occurrence of the reproductive and vegetative programs during the reproductive phase, results in the transformation of SMs into vegetative shoots.

In rice pla1, ectopic shoots eventually differentiate into small panicles with elongated bracts. In weak alleles of pla1, these flowers are apparently normal and set several seeds per panicle [18]. Although a small spike was observed in the tip of ectopic shoots in the fdk mutants, this was sterile (Figure 4B). Nevertheless, we conclude that wheat $f d k$ and rice pla 1 mutants show similar phenotypes. The pla mutation is controlled by at least three genes, PLA1, PLA2 and PLA3, which act independently [19-21]. These facts indicate that some common genetic cascades are involved in the phenotype of wheat $f d k$ and rice pla. The similar phenotypes of the $f d k$ with pla, especially pla1, suggest that PLA or related genes may be candidates for the $f d k$ mutation in wheat.

\section{Acknowledgements}

The authors thank Dr. Hidemi Kitano and Dr. Yutaka Okumoto for valuable suggestions. We are also grateful to the National Bioresource Project - Wheat (NBRPKOMUGI) for providing WT wheat strain. This experiment was performed at RIBF operated by the RIKEN Nishina Center and CNS, University of Tokyo. This work was supported in part by the Grant-in-Aid for Scientific Research on Innovative Areas from The Ministry of Education, Culture, Sports, Science and Technology (MEXT) KAKENHI Grant Number 24113517 to K. Murai, and the Japan Society for the Promotion of Science through the "Funding Program for Next Generation World-Leading Researchers," initiated by the Council for Science and Technology Policy to T. Abe. 


\section{REFERENCES}

[1] D. J. Miralles and G. A. Slafer, "Wheat Development," In: E. H. Satorre and G. A. Slafer, Eds., Wheat, Ecology and Physiology of Yield Determination, Food Products Press, New York, 1999, pp. 13-43.

[2] N. Shitsukawa, H. Kinjo, S. Takumi and K. Murai, "Heterochronic Development of the Floret Meristem Determines Grain Number per Spikelet in Diploid, Tetraploid and Hexaploid Wheats," Annals of Botany, Vol. 104, No. 2, 2009, pp. 243-251. http://dx.doi.org/10.1093/aob/mcp129

[3] H. Opik and S. Rolfe, "The Physiology of Flowering Plants," 4th Edition, Cambridge University Press, Cambridge, 2005.

http://dx.doi.org/10.1017/CBO9781139164450

[4] P. K. M. Hay and R. P. Ellis, "The Control of Flowering in Wheat and Barley: What Recent Advances in Molecular Genetics Can Reveal," Annals of Botany, Vol. 82, No. 5, 1998, pp. 541-554. http://dx.doi.org/10.1006/anbo.1998.0733

[5] R. J. Schmidt and B. A. Ambrose, "The Blooming of Grass Flower Development," Current Opinion in Plant Biology, Vol. 1, No. 1, 1998, pp. 60-67. http://dx.doi.org/10.1016/S1369-5266(98)80129-5

[6] P. Bommert, N. Satoh-Nagasawa, D. Jackson and H.-Y. Hirano, "Genetics and Evolution of Inflorescence and Flower Development in Grasses," Plant and Cell Physiology, Vol. 46, No. 1, 2005, pp. 69-78. http://dx.doi.org/10.1093/pcp/pci504

[7] K. Murai, S. Takumi, H. Koga and Y. Ogihara, "Pistillody, Homeotic Transformation of Stamens into Pistil-Like Structures, Caused by Nuclear-Cytoplasm Interaction in Wheat," The Plant Journal, Vol. 29, No. 2, 2002, pp. 169-181. http://dx.doi.org/10.1093/pcp/pci504

[8] N. Shitsukawa, A. Takagishi, C. Ikari, S. Takumi and K. Murai, "WFL, a Wheat FLORICAULA/LEAFY Ortholog, Is Associated with Spikelet Formation as Lateral Branch of the Inflorescence Meristem," Genes and Genetic System, Vol. 81, No. 1, 2006, pp. 13-20.

http://dx.doi.org/10.1266/ggs.81.13

[9] K. Ikeda, N. Nagasawa and Y. Nagato, "ABERRANT PANICLE ORGANIZATION 1 Temporally Regulates Meristem Identity in Rice," Developmental Biology, Vol. 282, No. 2, 2005, pp. 349-360. http://dx.doi.org/10.1016/j.ydbio.2005.03.016

[10] K. Ikeda, M. Ito, N. Nagasawa, J. Kyozuka and Y. Nagato, "Rice ABERRANT PANICLE ORGANIZATION 1, Encoding an F-Box Protein, Regulates Meristem Fate," The Plant Journal, Vol. 51, No. 6, 2007, pp. 1030-1040. http://dx.doi.org/10.1111/j.1365-313X.2007.03200.x

[11] K. Ikeda-Kawakatsu, N. Yasuno, T. Oikawa, S. Iida, Y. Nagato, M. Maekawa and J. Kyozuka, "Expression Level of ABERRANT PANICLE ORGANIZATION 1 Determines Rice Inflorescence form Through Control of Cell Proliferation in the Meristem," Plant Physiology, Vol. 150, No. 2, 2009, pp. 736-747.

http://dx.doi.org/10.1104/pp.109.136739
[12] X. Gao, W. Liang, C. Yin, S. Ji, H. Wang, X. Su, C. Guo, H. Kong, H. Xue and D. Zhang, "The SEPALLATA-Like Gene OsMADS34 Is Required for Rice Inflorescence and Spikelet Development," Plant Physiology, Vol. 153, No. 2, 2010, pp. 728-740. http://dx.doi.org/10.1104/pp.110.156711

[13] K. Kobayashi, M. Maekawa, A. Miyao, H. Hirochika and J. Kyozuka, "PANICLE PHYTOMER 2 (PAP2), Encoding a SEPALLATA Subfamily MADS-Box Protein, Positively Controls Spikelet Meristem Identity in Rice," Plant Cell Physiology, Vol. 51, No. 1, 2010, pp. 47-57. http://dx.doi.org/10.1093/pcp/pcp166

[14] K. Ikeda-Kawakatsu, M. Maekawa, T. Izawa, J.-I. Itoh and Y. Nagato, "ABERRANT PANICLE ORGANIZATION 2/RFL, the Rice Ortholog of Arabidopsis LEAFY, Suppresses The Transition from Inflorescence Meristem to Floral Meristem through Interaction with APO1," The Plant Journal, Vol. 69, No. 1, 2012, pp. 168-180. http://dx.doi.org/10.1111/j.1365-313X.2011.04781.x

[15] Y. Kazama, T. Hirano, H. Saito, Y. Liu, S. Ohbu, Y. Hayashi and T. Abe, "Characterization of Highly Efficient heavy-Ion Mutagenesis in Arabidopsis thaliana," BMC Plant Biology, Vol. 11, 2011, p. 161. http://dx.doi.org/10.1186/1471-2229-11-161

[16] Y. Kazama, M. T. Fujiwara, T. Hirano, S. Ohbu, H. Saito, H. Ichida, Y. Hayashi and T. Abe, "Characterization of a Heavy-Ion Induced White Flower Mutant of Allotetraploid Nicotiana tabacum," Plant Cell Report, Vol. 32, No. 1, 2013, pp. 11-19. http://dx.doi.org/10.1007/s00299-012-1336-7

[17] K. Murai, A. Nishiura, Y. Kazama and T. Abe, "A LargeScale Mutant Panel in Wheat Developed Using HeavyIon Beam Mutagenesis and Its Application to Genetic Research," Nuclear Instruments and Methods in Physics Research B: Beam Interactions with Materials and Atoms, Vol. 314, 2013, pp. 59-62. http://dx.doi.org/10.1016/j.nimb.2013.05.026

[18] J.-I. Itoh, J.-I., A. Hasegawa, H. Kitano and Y. Nagato, "A Recessive Heterochronic Mutation, plastochron 1, Shortens the Plastochron and Elongates the Vegetative Phase in Rice," The Plant Cell, Vol. 10, No. 9, 1998, pp. 1511-1521. http://dx.doi.org/10.1016/j.nimb.2013.05.026

[19] T. Kawakatsu, J.-I. Itoh, K. Miyoshi, N. Kurata, N. Alvarez, B. Veit and Y. Nagato, "PLASTOCHRON 2 Regulates Leaf Initiation and Maturation in Rice," The Plant Cell, Vol. 18, No. 3, 2006, pp. 612-625. http://dx.doi.org/10.1105/tpc.105.037622

[20] K. Kawakatsu, T., G. Taramino, J.-I. Itoh, J. Allen, Y. Sato, S.-K. Hong, R. Yule, et al., "PLASTOCHRON3/ GOLIATH Encodes a Glutamate Carboxypeptidase Required for Proper Development in Rice," The Plant Journal, Vol. 58, No. 6, 2009, pp. 1028-1040. http://dx.doi.org/10.1111/j.1365-313X.2009.03841.x

[21] K. Miyoshi, B.-O. Ahn, T. Kawakatsu, Y. Ito, J.-I. Itoh and Y. Nagato, "PLASTOCHRON 1, a Timekeeper of Leaf Initiation in Rice, Encodes Cytochrome P450," Proceedings of the National Academy of Science of the USA, Vol. 101, No. 3, 2004, pp. 875-880. http://dx.doi.org/10.1073/pnas.2636936100 
[22] B. Veit, S. P. Briggs, R. J. Schmidt, M. F. Yanofsky and S. Hake, "Regulation of Leaf Initiation by the terminal ear 1 Gene of Maize," Nature, Vol. 393, No. 6681, 1998, pp. 166-168. http://dx.doi.org/10.1038/30239

[23] C. A. Helliwell, A. N. Chin-Atkins, I. W. Wilson, R. Chapple, E. S. Dennis and A. Chaudhury, "The Arabidopsis AMP1 Gene Encodes a Putative Glutamate Carboxypeptidase," The Plant Cell, Vol. 13, No. 9, 2001, pp. 2115-2125.

[24] M, Komatsu, A. Chujo, Y. Nagato, K. Shimamoto and J. Kyozuka, "FRIZZY PANICLE Is Required to Prevent the Formation of Axillary Meristems and to Establish Floral Meristem Identity in Rice Spikelets," Development, Vol. 130, No. 16, 2003, pp. 3841-3850. http://dx.doi.org/10.1242/dev.00564

[25] N. Shitsukawa, C. Tahira, K.-I. Kassai, C. Hirabayashi, T. Shimizu, S. Takumi, K. Mochida, K. Kawaura, Y. Ogihara and K. Murai, "Genetic and Epigenetic Alteration among Three Homoeologous Genes of a Class E MADS Box Gene in Hexaploid Wheat," Plant Cell, Vol. 19, No.
6, 2007, pp. 1723-1737.

http://dx.doi.org/10.1105/tpc.107.051813

[26] K. J. Simons, J. P. Fellers, H. N. Trick, Z. Zhang, Y. S. Tai, B. S. Gill and J. D. Faris, "Molecular Characterization of the Major Wheat Domestication Gene Q," Genetics, Vol. 172, No. 1, 2006, pp. 547-555. http://dx.doi.org/10.1534/genetics.105.044727

[27] J.-I. Itoh, K.-I. Nonomura, K. Ikeda, S. Yamaki, Y. Inukai, H. Yamagishi, H. Kitano and Y. Nagato, "Rice Plant Development: From Zygote to Spikelet," Plant Cell Physiology, Vol. 46, No. 1, 2005, pp. 23-47. http://dx.doi.org/10.1093/pcp/pci501

[28] H. Kinjo, N. Shitsukawa, S. Takumi and K. Murai, "Diversification of Three APETALA1/FRUITFULL-Like Genes in Wheat," Molecular Genetics and Genomics, Vol. 287, No. 4, 2012, pp. 283-294.

http://dx.doi.org/10.1007/s00438-012-0679-7 